\title{
THE GLOBAL CAUCHY PROBLEM FOR THE NLS WITH HIGHER ORDER ANISOTROPIC DISPERSION
}

\author{
LEONID CHAICHENETS and NIKOLAOS PATTAKOS \\ Department of Mathematics, Institute for Analysis, Karlsruhe Institute of Technology, 76128 Karlsruhe, \\ Germany \\ e-mails:leonid.chaichenets@kit.edu; nikolaos.pattakos@kit.edu
}

(Received 1 April 2019; revised 18 November 2019; accepted 18 November 2019; first published online 12 December 2019)

\begin{abstract}
We use a method developed by Strauss to obtain global well-posedness results in the mild sense and existence of asymptotic states for the small data Cauchy problem in modulation spaces $M_{p, q}^{s}\left(\mathbb{R}^{d}\right)$, where $q=1$ and $s \geq 0$ or $q \in(1, \infty]$ and $s>\frac{d}{q^{\prime}}$ for a nonlinear Schrödinger equation with higher order anisotropic dispersion and algebraic nonlinearities.
\end{abstract}

2010 Mathematics Subject Classification. Primary 35A01, 35A02, 35Q55; Secondary 35B40.

1. Introduction and main results. We are interested in the following Cauchy problem

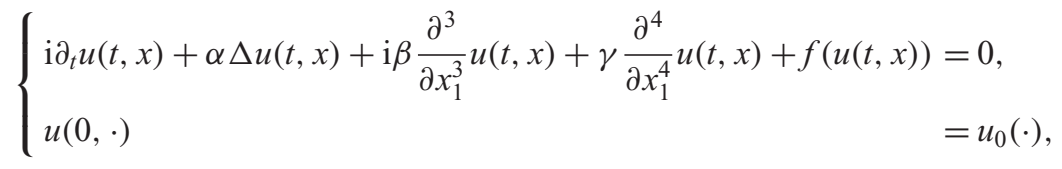

where $(t, x)=\left(t, x_{1}, x^{\prime}\right) \in \mathbb{R} \times \mathbb{R} \times \mathbb{R}^{d-1}, d \geq 2, \alpha \in \mathbb{R} \backslash\{0\}$, and $(\beta, \gamma) \in \mathbb{R}^{2} \backslash\{(0,0)\}$. The NLS with higher order dispersion was introduced by Karpman, see [9] and the references therein. It has applications in the context of high-speed soliton transmission in long-haul optical communication systems, see [6] and [8]. The case where the coefficiets $\alpha, \beta, \gamma$ are time-dependent has been studied in [3] in one dimension for the cubic nonlinearity, $f(u)=|u|^{2} u$, with initial data in $L^{2}(\mathbb{R})$-based Sobolev spaces. In [2], it is proved that (1.1) with nonlinearity $f(u)=|u|^{p} u$ where

$$
p< \begin{cases}\frac{4}{d-\frac{1}{2}}, & \gamma \neq 0, \\ \frac{4}{d-\frac{1}{3}}, & \gamma=0,\end{cases}
$$

is globally well-posed in $L^{2}\left(\mathbb{R}^{d}\right)$ via Strichartz estimates and the charge conservation equation

$$
\|u(t, \cdot)\|_{L^{2}\left(\mathbb{R}^{d}\right)}=\left\|u_{0}\right\|_{L^{2}\left(\mathbb{R}^{d}\right)}, \quad \forall t \in \mathbb{R} .
$$

In the same paper, the case of initial data $u_{0} \in H_{a}^{1}\left(\mathbb{R}^{d}\right)$ is studied where

$$
H_{a}^{1}\left(\mathbb{R}^{d}\right):=\left\{u \in L^{2}\left(\mathbb{R}^{d}\right) \mid \nabla u, \partial_{x_{1}}^{2} u \in L^{2}\left(\mathbb{R}^{d}\right)\right\}
$$


is equipped with the norm

$$
\|u\|_{H_{a}^{1}\left(\mathbb{R}^{d}\right)}:=\left(\|u\|_{L^{2}\left(\mathbb{R}^{d}\right)}^{2}+\|\nabla u\|_{L^{2}\left(\mathbb{R}^{d}\right)}^{2}+\left\|\partial_{x_{1}}^{2} u\right\|_{L^{2}\left(\mathbb{R}^{d}\right)}^{2}\right)^{\frac{1}{2}} .
$$

In this paper, we consider the Cauchy problem (1.1) with initial data $u_{0}$ in modulation spaces $M_{p, q}^{s}\left(\mathbb{R}^{d}\right)$. Modulation spaces were introduced by Feichtinger in [7] and since then, they have become canonical for both time frequency and phase space analysis. They provide an excellent substitute for estimates that are known to fail on Lebesgue spaces. To state the definition of a modulation space, we need to fix some notation. We will denote by $S^{\prime}\left(\mathbb{R}^{d}\right)$ the space of tempered distributions. Let $Q_{0}$ be the unit cube with center the origin in $\mathbb{R}^{d}$ and its translations $Q_{k}:=Q_{0}+k$ for all $k \in \mathbb{Z}^{d}$. Consider a partition of unity $\left\{\sigma_{k}=\sigma_{0}(\cdot-k)\right\}_{k \in \mathbb{Z}^{d}} \subset C^{\infty}\left(\mathbb{R}^{d}\right)$ satisfying

- $\exists c>0: \forall \eta \in Q_{0}:\left|\sigma_{0}(\eta)\right| \geq c$,

- $\operatorname{supp}\left(\sigma_{0}\right) \subseteq\left\{\xi \in \mathbb{R}^{d}:|\xi|<\sqrt{d}\right\}=: B(0, \sqrt{d})$,

and define the isometric decomposition operators

$$
\square_{k}:=\mathcal{F}^{(-1)} \sigma_{k} \mathcal{F}, \quad \forall k \in \mathbb{Z}^{d},
$$

where $\mathcal{F}$ denotes the Fourier transform in $\mathbb{R}^{d}$. Then, the norm of a tempered distribution $f \in S^{\prime}\left(\mathbb{R}^{d}\right)$ in the modulation space $M_{p, q}^{s}\left(\mathbb{R}^{d}\right)$, where $s \in \mathbb{R}, 1 \leq p, q \leq \infty$, is given by

$$
\|f\|_{M_{p, q}^{s}}:=\left\|\left\{\langle k\rangle^{s}\left\|\square_{k} f\right\|_{p}\right\}_{k \in \mathbb{Z}^{d}}\right\|_{l^{q}\left(\mathbb{Z}^{d}\right)},
$$

where we denote by $\langle k\rangle=1+|k|$ the Japanese bracket. It can be proved that different choices of the function $\sigma_{0}$ lead to equivalent norms in $M_{p, q}^{s}\left(\mathbb{R}^{d}\right)$ (see e.g. [4, Proposition 2.9]). When $s=0$, we denote the space $M_{p, q}^{0}\left(\mathbb{R}^{d}\right)$ by $M_{p, q}\left(\mathbb{R}^{d}\right)$. In the special case where $p=q=2$, we have $M_{2,2}^{s}\left(\mathbb{R}^{d}\right)=H^{s}\left(\mathbb{R}^{d}\right)$ the usual Sobolev spaces.

For $\alpha \in \mathbb{R}$, we define the weighted mixed-norm space

$$
L_{\alpha}^{\infty}\left(\mathbb{R}, M_{p, q}^{s}\left(\mathbb{R}^{d}\right)\right):=\left\{u \in L^{\infty}\left(\mathbb{R}, M_{p, q}^{s}\left(\mathbb{R}^{d}\right)\right) \mid\|u\|_{L_{\alpha}^{\infty}\left(\mathbb{R}, M_{p, q}^{s}\left(\mathbb{R}^{d}\right)\right)}<\infty\right\},
$$

where

$$
\|u\|_{L_{\alpha}^{\infty}\left(\mathbb{R}, M_{p, q}^{s}\left(\mathbb{R}^{d}\right)\right)}:=\sup _{t \in \mathbb{R}}\langle t\rangle^{\alpha}\|u(t, \cdot)\|_{M_{p, q}^{s}} .
$$

Let us denote by $\pi\left(u^{m+1}\right)$ any $(m+1)$-time product of $u$ and $\bar{u}$, where $m \in \mathbb{Z}_{+}$. Define also the quantity

$$
\frac{2}{\gamma_{m, d}}= \begin{cases}\left(d-\frac{1}{2}\right)\left(\frac{m}{2(m+2)}\right), & \gamma \neq 0, \\ \left(d-\frac{1}{3}\right)\left(\frac{m}{2(m+2)}\right), & \gamma=0 .\end{cases}
$$

Futhermore, let $m_{0}$ denote the positive root of

$$
\begin{cases}(2 d-1) x^{2}+(2 d-5) x-8=0, & \gamma \neq 0 \\ (3 d-1) x^{2}+(3 d-7) x-12=0, & \gamma=0\end{cases}
$$

The main results are the following theorems. 
THEOREM 1. Suppose that $d \geq 1, f(u)= \pm \pi\left(u^{m+1}\right), m \in \mathbb{Z}_{+}$with $m>m_{0}$ and $q \in$ $[1, \infty]$. For $q=1$, let $s \geq 0$ and for $q>1$, let $s>\frac{d}{q^{\prime}}$. Then there exists a $\delta>0$ such that for any $u_{0} \in M_{\frac{m+2}{m+1}, q}^{s}\left(\mathbb{R}^{d}\right)$ with $\left\|u_{0}\right\|_{M_{\frac{m+2}{m+1}, q}^{s}} \leq \delta$, the Cauchy problem (1.1) admits a unique global solution

$$
u \in L_{\frac{2}{\gamma_{m, d}}}^{\infty}\left(\mathbb{R}, M_{2+m, q}^{s}\left(\mathbb{R}^{d}\right)\right)
$$

The restriction on the power of the nonlinearity described in Theorem 1 is explained in remark 11 .

THEOREM 2. Suppose that $d \geq 2, f(u)=\lambda\left(e^{\rho|u|^{2}}-1\right) u, \lambda \in \mathbb{C}$ and $\rho>0$. In addition, let $s \geq 0$ if $q=1$ and let $s>\frac{d}{q^{\prime}}$ if $q \in(1, \infty]$. There exists $\delta>0$ such that for any $u_{0} \in$ $M_{\frac{4}{3}, q}^{s}\left(\mathbb{R}^{d}\right)$ with $\left\|u_{0}\right\|_{M_{\frac{4}{3}, q}^{s}} \leq \delta$, the Cauchy problem (1.1) admits a unique global solution $u$ in the space $L_{\frac{2}{\gamma_{2, d}}}^{\infty}\left(\mathbb{R}, M_{4, q}^{s}\left(\mathbb{R}^{d}\right)\right)$.

REMARK 3. For $q<\infty$, the solution from Theorems 1 and 2 is a continuous function with values in the corresponding modulation space, that is, indeed a mild solution. For the more delicate situation $q=\infty$, see [12].

The existence of the asymptotic states is stated in the following proposition. Notice that the solution $u$ obtained in Theorem 1 lies in $M_{2, q}^{s}\left(\mathbb{R}^{d}\right)$, too (see (2.22)).

PROPOSITION 4. Under the assumptions of Theorem 1, the global solution u scatters to

$$
u_{+}:=u_{0} \pm \mathrm{i} \int_{0}^{\infty} W(-\tau)\left(\pi\left(u^{m+1}\right)\right) d \tau
$$

in $M_{2, q}^{s}\left(\mathbb{R}^{d}\right)$, that is,

$$
\lim _{t \rightarrow \infty}\left\|u(t)-W(t) u_{+}\right\|_{M_{2, q}^{s}}=0
$$

A corresponding statement is true for the limit $t \rightarrow-\infty$. Also, a similar result holds in the situation of Theorem 2.

REMARK 5. Let us mention that using the same techniques, we also obtain a weak version of asymptotic completeness, that is, if $u_{+} \in M_{\frac{m+2}{m+1}, q}^{s}\left(\mathbb{R}^{d}\right)$ and has sufficiently small norm, then there exists a unique global solution $u \in L_{\frac{2}{\gamma_{m, d}}}^{\infty+}\left(\mathbb{R}, M_{m+2, q}^{s}\left(\mathbb{R}^{d}\right)\right)$ such that (1.8) holds.

Asymptotic completeness, for the higher order NLS with anisotropic dispersion in the classical setting of weighted $L^{2}$ spaces, has been studied in [14].

The idea of studying the Cauchy problem (1.1) with such time-decay norm is inspired by [17], where the authors considered the NLS and the NLKG equations. As mentioned there, this idea goes back to the work of Strauss, see [15]. Their results were improved in $[\mathbf{1 0}]$ and $[\mathbf{1 1}]$ where the author considered the nonlinear higher order Schrödinger equation

$$
\mathrm{i} \partial_{t} u+\phi(\sqrt{-\Delta}) u=f(u)
$$

where $\phi(\sqrt{-\Delta})=\mathcal{F}^{-1} \phi(|\xi|) \mathcal{F}$ and $\phi$ is a polynomial, with initial data $u_{0}$ in a modulation space. 
REMARK 6. Notice that Theorem 1 does not include the cubic nonlinearity in dimension $d=1$ since $m$ has to be strictly bigger than $m_{0}$ which is the positive root of the quadratics in (1.5), that is, $m_{0}=\frac{3+\sqrt{41}}{2}$, if $\gamma \neq 0$ and $m_{0}=\frac{4+\sqrt{110}}{4}$, if $\gamma=0$. In both cases, $m_{0}>3$.

REMARK 7. In Ref. [17, Theorems 1.1 and 1.2], the authors only considered modulation spaces $M_{p, q}^{s}\left(\mathbb{R}^{d}\right)$ with $q=1$. But, by Theorem 8 , their crucial estimate (6.6) also holds for $q \in(1, \infty]$ and $s>\frac{d}{q^{\prime}}$. Hence, the statements of their theorems are true in this case, too.

Moreover, the generalizations of Ref. [17, Theorems 1.3 and 1.4] from $M_{2,1}\left(\mathbb{R}^{d}\right)$ to $M_{2, q}^{s}\left(\mathbb{R}^{d}\right)$ and to the case of the higher order NLS shall be considered by the authors in a future work.

1.1. Preliminaries. It is known that for $s>d / q^{\prime}$ (where $q^{\prime}$ is the conjugate exponent of $q$ ) and $p, q \in[1, \infty]$, the embedding

$$
M_{p, q}^{s}\left(\mathbb{R}^{d}\right) \hookrightarrow C_{\mathrm{b}}\left(\mathbb{R}^{d}\right)=\left\{f: \mathbb{R}^{d} \rightarrow \mathbb{C} \mid f \text { continuous and bounded }\right\}
$$

is continuous. The same is true for the embedding

$$
M_{p_{1}, q_{1}}^{s_{1}}\left(\mathbb{R}^{d}\right) \hookrightarrow M_{p_{2}, q_{2}}^{s_{2}}\left(\mathbb{R}^{d}\right),
$$

which holds for any $s_{1}, s_{2} \in \mathbb{R}$ and any $p_{1}, p_{2}, q_{1}, q_{2} \in[1, \infty]$ satisfying $p_{1} \leq p_{2}$ and either

$$
\begin{aligned}
& q_{1} \leq q_{2} \text { and } \quad s_{1} \geq s_{2} \\
& \quad \text { or } \\
& q_{2}<q_{1} \text { and } s_{1}>s_{2}+\frac{d}{q_{2}}-\frac{d}{q_{1}} .
\end{aligned}
$$

(see [7, Propositions 6.5 and 6.8]).

We are going to use the following Hölder-type inequality for modulation spaces which appeared in Ref. [4, Theorem 4.3] (see also [5]).

THEOREM 8. Let $d \geq 1$ and $1 \leq p, p_{1}, p_{2}, q \leq \infty$ such that $\frac{1}{p}=\frac{1}{p_{1}}+\frac{1}{p_{2}}$. For $q=1$, let $s \geq 0$ and for $q \in(1, \infty]$ let $s>\frac{d}{q^{\prime}}$. Then there exists a constant $C=C(d, s, q)>0$ such that

$$
\|f g\|_{M_{p, q}^{s}} \leq C\|f\|_{M_{p_{1}, q}^{s}}\|g\|_{M_{p_{2}, q}^{s}},
$$

for all $f \in M_{p_{1}, q}^{s}\left(\mathbb{R}^{d}\right)$ and $g \in M_{p_{2}, q}^{s}\left(\mathbb{R}^{d}\right)$.

The propagator of the homogeneous Schrödigner equation with higher order anisotropic dispersion is given by

$$
W(t)=\mathcal{F}^{(-1)} e^{\mathrm{i}\left(\alpha|\xi|^{2}+\beta \xi_{1}^{3}+\gamma \xi_{1}^{4}\right) t} \mathcal{F},
$$

where $\xi=\left(\xi_{1}, \xi^{\prime}\right) \in \mathbb{R} \times \mathbb{R}^{d-1}$. For the rest of the paper, $A \lesssim B$ shall mean that there is a constant $C>0$ such that $A \leq C B$. The next dispersive estimate, in the isotropic case $\beta=0$, has been first proven in [1]. The anisotropic scenario which we are interested in is from Ref. [2, Theorem 1.1]:

Theorem 9. Consider $p \in[2, \infty]$ and $f \in L^{p^{\prime}}\left(\mathbb{R}^{d}\right)$. Then

$$
\|W(t) f\|_{L^{p}\left(\mathbb{R}^{d}\right)} \lesssim|t|^{-\mu}\|f\|_{L^{p^{\prime}}\left(\mathbb{R}^{d}\right)},
$$


where

$$
\mu=\mu(d, \gamma, p):= \begin{cases}\left(d-\frac{1}{2}\right)\left(\frac{1}{2}-\frac{1}{p}\right) & \gamma \neq 0, \\ \left(d-\frac{1}{3}\right)\left(\frac{1}{2}-\frac{1}{p}\right) & \gamma=0,\end{cases}
$$

and the implicit constant is independent of the function $f$ and the time $t$.

Using this, we claim the following

TheOREM 10. Consider $s \in \mathbb{R}, p \in[2, \infty]$ and $q \in[1, \infty]$. Then

$$
\|W(t) f\|_{M_{p, q}^{s}\left(\mathbb{R}^{d}\right)} \lesssim\langle t\rangle^{-\mu}\|f\|_{M_{p^{\prime}, q}^{s}\left(\mathbb{R}^{d}\right)},
$$

where $\mu=\mu(d, \gamma, p)$ is as in equation (1.14) and the implicit constant is independent of the function $f$ and the time $t$.

Proof. The operators $\square_{k}$ and $W(t)$ commute and hence we immediately arrive at

$$
\left\|\square_{k} W(t) f\right\|_{L^{p}\left(\mathbb{R}^{d}\right)} \lesssim|t|^{-\mu}\left\|\square_{k} f\right\|_{L^{p^{\prime}\left(\mathbb{R}^{d}\right)}} \quad \forall k \in \mathbb{Z}^{d} \forall t \in \mathbb{R} \backslash\{0\},
$$

by invoking Theorem 9. Moreover, as $p \in[2, \infty]$, we have

$$
\left\|\square_{k} W(t) f\right\|_{L^{p}\left(\mathbb{R}^{d}\right)} \lesssim\left\|\sigma_{k} e^{\mathrm{i}\left(\alpha|\xi|^{2}+\beta \xi_{1}^{3}+\gamma \xi_{1}^{4}\right) t} \hat{f}\right\|_{L^{p^{\prime}\left(\mathbb{R}^{d}\right)}} \lesssim\left\|\sigma_{k} \hat{f}\right\|_{L^{p}\left(\mathbb{R}^{d}\right)} \lesssim\left\|\square_{k} f\right\|_{L^{p^{\prime}}\left(\mathbb{R}^{d}\right),}
$$

for any $k \in \mathbb{Z}^{d}$ and any $t \in \mathbb{R}$. Above, we used the Hausdoff-Young inequality for the first and last estimate and the fact that $\operatorname{supp}\left(\sigma_{k}\right) \subseteq B_{\sqrt{d}}(k)$ for the second inequality. Taking the minimum of the right-hand sides of (1.16) and (1.17) shows

$$
\left\|\square_{k} W(t) f\right\|_{L^{p}\left(\mathbb{R}^{d}\right)} \lesssim\langle t\rangle^{-\mu}\left\|\square_{k} f\right\|_{L^{p^{\prime}}\left(\mathbb{R}^{d}\right)} \quad \forall k \in \mathbb{Z}^{d} \forall t \in \mathbb{R} .
$$

Multiplying by the weight $\langle k\rangle^{s}$ and taking the $l^{q}\left(\mathbb{Z}^{d}\right)$-norm on both sides, we arrive at the desired estimate.

2. Proofs of the main results. In this section, we present the proofs of the main theorems and Proposition 4.

Proof of Theorem 1. For the sake of brevity, let us shorten the notation by setting

$$
\|u\|:=\|u\|_{L_{\frac{2}{\gamma_{m, d}}}^{\infty}}\left(\mathbb{R}, M_{m+2, q}^{s}\left(\mathbb{R}^{d}\right)\right) .
$$

By the Banach fixed-point theorem, it suffices to show that the operator defined by

$$
\mathcal{T} u:=W(t) u_{0} \pm i \int_{0}^{t} W(t-\tau)\left(\pi\left(u^{m+1}\right)\right) \mathrm{d} \tau
$$

is a contractive self-mapping of the complete metric space

$$
M(R)=\left\{u \in L_{\frac{2}{\gamma_{m, d}}}^{\infty}\left(\mathbb{R}, M_{m+2, q}^{s}\left(\mathbb{R}^{d}\right)\right) \mid\|u\| \leq R\right\},
$$

for some $R \in \mathbb{R}_{+}$. We begin with the self-mapping property and observe that

$$
\|\mathcal{T} u\| \leq\left\|W(t) u_{0}\right\|+\left\|\int_{0}^{t} W(t-\tau)\left(\pi\left(u^{m+1}\right)\right) \mathrm{d} \tau\right\| .
$$


Notice, that $\mu(d, \gamma, m+2)=\frac{2}{\gamma_{m, d}}$ and hence, by the dispersive estimate (1.15), one obtains

$$
\left\|W(t) u_{0}\right\|=\sup _{t \in \mathbb{R}}\left[\langle t\rangle^{\frac{2}{\gamma_{m, d}}}\left\|W(t) u_{0}\right\|_{M_{m+2, q}^{s}}\right] \lesssim\left\|u_{0}\right\|_{M_{\frac{m+2}{m+q}, q}^{s}} .
$$

Introducing the smallness condition

$$
\left\|u_{0}\right\|_{M_{\frac{m+2}{m+1}, q}^{s}\left(\mathbb{R}^{d}\right)} \lesssim \frac{R}{2}
$$

leads to $\left\|W(t) u_{0}\right\| \leq \frac{R}{2}$.

For the integral term, we have the upper bound

$$
\sup _{t \in \mathbb{R}}\left[\langle t\rangle^{\frac{2}{\gamma_{m, d}}} \int_{0}^{t}\langle t-\tau\rangle^{-\frac{2}{\gamma_{m, d}}}\left\|\pi\left(u^{m+1}\right)\right\|_{M_{\frac{m+2}{m+1}, q}^{s}} \mathrm{~d} \tau\right] .
$$

Hölder's inequality for modulation spaces from Theorem 8 is applicable (due to the assumptions on $s, q$ ) and yields

$$
\left\|\pi\left(u^{m+1}\right)\right\|_{M_{\frac{m+2}{m+1}, q}^{s}} \lesssim\|u\|_{M_{m+2, q}^{s}}^{m+1} .
$$

Furthermore, as $u \in M(R)$, one has

$$
\|u(\tau, \cdot)\|_{M_{m+2, q}^{s}} \leq\langle\tau\rangle^{-\frac{2}{\gamma_{m, d}}}\|u\| \leq\langle\tau\rangle^{-\frac{2}{\gamma_{m, d}}} R \quad \forall \tau \in \mathbb{R}
$$

and we obtain the upper bound for the integral term

$$
R^{m+1} \sup _{t \in \mathbb{B}}\left[\langle t\rangle^{\frac{2}{\gamma_{m, d}}} \int_{0}^{t}\langle t-\tau\rangle^{-\frac{2}{\gamma_{m, d}}}\langle\tau\rangle^{-\frac{2(m+1)}{\gamma_{m, d}}} \mathrm{~d} \tau\right] .
$$

To be able to control the individual factors of the integral, we split it into $\int_{0}^{t}=\int_{0}^{\frac{t}{2}}+\int_{\frac{t}{2}}^{t}$. For the first summand, we have

$$
\begin{aligned}
\int_{0}^{\frac{t}{2}}\langle t-\tau\rangle^{-\frac{2}{\gamma_{m, d}}}\langle\tau\rangle^{-\frac{2(m+1)}{\gamma_{m, d}}} d \tau & \lesssim\left\langle\frac{t}{2}\right\rangle^{-\frac{2}{\gamma_{m, d}}} \frac{1}{1-\frac{2}{\gamma_{m, d}}(m+1)}\left(\left\langle\frac{t}{2}\right\rangle^{1-\frac{2}{\gamma_{m, d}}(m+1)}-1\right) \\
& \lesssim\langle t\rangle^{-\frac{2}{\gamma_{m, d}}}
\end{aligned}
$$

where we used the monotonicity of $\langle\cdot\rangle$ and the assumption $m>m_{0}$, which implies $\frac{2(m+1)}{\gamma_{m, d}}>$ 1. We similarly estimate the second summand by

$$
\int_{\frac{t}{2}}^{t}\langle t-\tau\rangle^{-\frac{2}{\gamma_{m, d}}}\langle\tau\rangle^{-\frac{2(m+1)}{\gamma_{m, d}}} d \tau \lesssim\langle t\rangle^{-\frac{2(m+1)}{\gamma_{m, d}}} \int_{\frac{t}{2}}^{t}\langle t-\tau\rangle^{-\frac{2}{\gamma_{m, d}}} \lesssim\langle t\rangle^{-\frac{2}{\gamma_{m, d}}} .
$$

Putting everything together, we arrive at the condition

$$
\|\mathcal{T} u\| \lesssim \frac{R}{2}+R^{m+1} \stackrel{!}{\leq} R
$$

which is satisfied for sufficiently small $R$. Similarly, we obtain

$$
\|\mathcal{T} u-\mathcal{T} v\| \lesssim\left(\|u\|^{m}+\|v\|^{m}\right)\|u-v\| \leq 2 R^{m}\|u-v\| .
$$

Hence, under a possibly smaller choice of $R$, the operator $\mathcal{T}$ is a contraction and the proof is complete. 
REMARK 11. Observe that the restriction $m>m_{0}$ corresponds to the boundedness of the terms in (2.10) and (2.11).

Proof of Theorem 2. As in the proof of Theorem 1, we shorten the notation of the norm by

$$
\|u\|:=\|u\|_{L_{\frac{2}{\gamma_{2, d}}}^{\infty}}\left(\mathbb{R}, M_{4, q}^{s}\right)
$$

and introduce the operator

$$
\mathcal{T} u=W(t) u_{0} \pm \mathrm{i} \int_{0}^{t} W(t-\tau)(f(u)) \mathrm{d} \tau,
$$

which we want to be a contractive self-mapping of the complete metric space $M(R)$ for some $R \in \mathbb{R}_{+}$. We begin with the self-mapping property. By the definition of the nonlinearity $f(u)=\lambda\left(e^{\rho|u|^{2}}-1\right) u$, we have

$$
f(u)=\lambda \sum_{k=1}^{\infty} \frac{\rho^{k}}{k !}|u|^{2 k} u .
$$

Following the proof of Theorem 1, we arrive at

$$
\|\mathcal{T} u\| \lesssim\left\|u_{0}\right\|_{M_{\frac{4}{3}, q}^{s}}+\sum_{k=1}^{\infty} \sup _{t \in \mathbb{R}}\left[\langle t\rangle^{\frac{2}{\gamma_{2, d}}} \int_{0}^{t}\langle t-\tau\rangle^{-\frac{2}{\gamma_{2, d}}} \frac{\rho^{k}}{k !}\left\||u|^{2 k} u\right\|_{M_{\frac{4}{3}, q}^{s}} \mathrm{~d} \tau\right] .
$$

Hölder's inequality for modulation spaces from Theorem 8 is applicable (due to the assumptions on $s, q$ ) and yields the estimate

$$
\left\||u|^{2 k} u\right\|_{M_{\frac{4}{3}, q}^{s}} \lesssim\|u\|_{M_{4, q}^{s}}^{3}\|u\|_{M_{\infty, q}^{s}}^{2 k-2} \lesssim\|u\|_{M_{4, q}^{s}}^{2 k+1},
$$

where in the second inequality we used (1.11), that is, the embedding $M_{4, q}^{s}\left(\mathbb{R}^{d}\right) \hookrightarrow$ $M_{\infty, q}^{s}\left(\mathbb{R}^{d}\right)$. Hence, by (2.8) for $m=2$, we obtain

$$
\|\mathcal{T} u\| \lesssim\left\|u_{0}\right\|_{M_{\frac{4}{3}, q}^{s}}+\sum_{k=1}^{\infty} \frac{\rho^{k}}{k !} R^{2 k+1} \sup _{t \in \mathbb{R}}\left[\langle t\rangle^{\frac{2}{\gamma_{2, d}}} \int_{0}^{t}\langle t-\tau\rangle^{-\frac{2}{\gamma_{2, d}}}\langle\tau\rangle^{-(2 k+1) \frac{2}{\gamma_{2, d}}} \mathrm{~d} \tau\right] .
$$

The supremum above is finite by the same reasoning as in the proof of Theorem 1 and we therefore arrive at the condition

$$
\|\mathcal{T} u\| \lesssim\left\|u_{0}\right\|_{M_{\frac{4}{3}, q}^{s}}+\sum_{k=1}^{\infty} \frac{\rho^{k}}{k !} R^{2 k+1}=\left\|u_{0}\right\|_{M_{\frac{4}{3}, q}^{s}}+\left(R e^{\rho R^{2}}-1\right) \stackrel{!}{\leq} R .
$$

Thus, if $\left\|u_{0}\right\|_{M_{\frac{4}{3}, q}^{s}} \lesssim \frac{R}{2}$ and $R>0$ is sufficiently small, the operator $\mathcal{T}$ is a self-mapping of the space $M(R)$. The contraction property is proved in a similar way.

Proof of Proposition 4. We only consider the case $t \rightarrow \infty$ and the power nonlinearity $\pi\left(u^{m+1}\right)$. Observe that

$$
\begin{aligned}
\left\|u(t)-W(t) u_{1}\right\|_{M_{2, q}^{s}} & =\left\|W(-t) u(t)-u_{1}\right\|_{M_{2, q}^{s}} \\
& =\left\|u_{0}-u_{1} \pm \mathrm{i} \int_{0}^{t} W(-\tau)\left(\pi\left(u^{m+1}\right)\right) d \tau\right\|_{M_{2, q}^{s}},
\end{aligned}
$$


for any $u_{1} \in M_{2, q}^{s}\left(\mathbb{R}^{d}\right)$. Thus, $\lim _{t \rightarrow \infty}\left\|u(t)-W(t) u_{1}\right\|_{M_{2, q}^{s}}=0$ iff the integral

$$
\int_{0}^{\infty} W(-\tau)\left(\pi\left(u^{m+1}\right)\right) d \tau
$$

converges conditionally in $M_{2, q}^{s}\left(\mathbb{R}^{d}\right)$ and $u_{1}=u_{+}$. Indeed, using that $W(t)$ is an isometry on $M_{2, q}^{s}\left(\mathbb{R}^{d}\right)$, the embedding $M_{\frac{m+2}{m+1}, q}^{s}\left(\mathbb{R}^{d}\right) \hookrightarrow M_{2, q}^{s}\left(\mathbb{R}^{d}\right)$ and Hölder's inequality from Theorem 8 , we obtain

$$
\begin{aligned}
\left\|\int_{0}^{\infty} W(-\tau)\left(\pi\left(u^{m+1}\right)\right) d \tau\right\|_{M_{2, q}^{s}} & \leq \int_{0}^{\infty}\left\|W(-\tau)\left(\pi\left(u^{m+1}\right)\right)\right\|_{M_{2, q}^{s}} d \tau \\
& =\int_{0}^{\infty}\left\|\left(\pi\left(u^{m+1}\right)\right)\right\|_{M_{2, q}^{s}} d \tau \\
& \leq \int_{0}^{\infty}\left\|\left(\pi\left(u^{m+1}\right)\right)\right\|_{M_{\frac{m+2}{m+1}, q}^{s}} d \tau \\
& \leq \int_{0}^{\infty}\|u\|_{M_{m+2, q}^{s+1}}^{m+1} d \tau \\
& \leq\|u\|_{L_{\frac{2}{\gamma_{m, d}}}^{\infty}}\left(\mathbb{R}, M_{m+2, q}^{s}\right) \int_{0}^{\infty}\langle\tau\rangle^{-\frac{2}{\gamma_{m, d}}(m+1)} d \tau .
\end{aligned}
$$

The last integral is finite since $\frac{2(m+1)}{\gamma_{m, d}}>1$ and hence the integral in (2.21) converges absolutely.

ACKNOWLEDGments. The authors would like to thank the anonymous referee and Professor Jean-Claude Saut for their fruitful comments.

Funded by the Deutsche Forschungsgemeinschaft (DFG, German Research Foundation) - project-id 258734477 - SFB 1173.

\section{REFERENCES}

1. M. Ben-Artzi, H. Koch and J.-C. Saut, Dispersion estimates for fourth order Schrödinger equations. C. R. Acad. Sci. Paris, Sér. I Math. 330 (2000), 87-92.

2. O. Bouchel, Remarks on NLS with higher order anisotropic dispersion. Adv. Differential Equ. 13(1-2) (2008), 169-198.

3. X. Carvajal, M. Panthee and M. Scialom, On well-posedness of the third order NLS equation with time dependent coefficients. Commun. Contemp. Math. (2015). DOI: 10.1142/ S021919971450031X.

4. L. Chaichenets, Modulation spaces and nonlinear Schrödinger equations. PhD Thesis (Karlsruhe Institute of Technology, 2018). DOI: 10.5445/IR/1000088173.

5. L. Chaichenets, D. Hundertmark, P. Kunstmann and N. Pattakos, Local wellposedness for the nonlinear Schrödinger equation in modulation spaces $M_{p, q}^{s}\left(\mathbb{R}^{d}\right)$. preprint (2016), arXiv: 1610.08298.

6. F. J. Diaz-Ortero and P. Chamorro-Posada, Interchannel soliton collisions in periodic dispersion maps on the presence of third order dispersion. J. Nonlinear Math. Phys. 15 (2008), $137-143$.

7. H. G. Feichtinger, Modulation spaces on locally compact Abelian groups. Technical Report, University of Vienna, 1983, in Proceedings of the International Conference on Wavelet and Applications (New Delhi Allied Publishers, India, 2003), 99-140.

8. M. Karlsson and A. Höök, Soliton-like pulses governed by fourth order dispersion in optical fibers. Opt. Commun. 104 (1994), 303-307. 
9. V.I. Karpman, Stabilization of soliton instabilities by higher-order dispersion: Fourth order nonlinear Schrödinger-type equations. Phys. Rev. E 53(2) (1996), R1336-R1339.

10. T. Kato, The global Cauchy problems for the nonlinear dispersive equations on modulation spaces. J. Math. Anal. Appl. 413 (2014), 821-840.

11. T. Kato, Solutions to nonlinear higher order Schrödinger equations with small initial data on modulation spaces. Adv. Differential Equations 21 (2016), 201-234.

12. P. Kunstmann, Modulation type spaces for generators of polynomially bounded groups and Schrödinger equations. Semigroup Forum, (2019). DOI: 10.1007/s00233-019-10016-1.

13. M. Ruzhansky and T. Ville, Pseudo-Differential Operators and Symmetries, vol. 2, PseudoDifferential Operators (Birkhäuser, Basel, 2010), ISBN 978-3-7643-8513-2.

14. J.-C. Saut and J.-I. Segata, Asymptotic behavior in time of solution to the nonlinear Schrödinger equation with higher order anisotropic dispersion. Discrete Contin. Dyn. Syst. 39 (2019), 219-239.

15. W. Strauss, Nonlinear scattering theory at low energy. J. Funct. Anal. 41 (1981), 110-133.

16. M. Sugimoto, N. Tomita and B. Wang, Remarks on nonlinear operations on modulation spaces. Integr. Transf. Spec. F. 22(4-5) (2011), 351-358.

17. B. X. Wang and H. Hudzik, The global Cauchy problem for the NLS and NLKG with small rough data. J. Differ. Eq. 232 (2007), 36-73. 